\title{
CH CYGNI HALF a CENTURY AGO - CHANGING ACTIVITY OF THE COOL COMPONENT
}

\author{
Władysław Dziewulski*, \\ Marek Muciek, Maciej Mikołajewski \\ Institute of Astronomy, \\ ul. Chopina $12 / 18$, PL-87100 TORUN, Poland
}

$\mathrm{CH}$ Cyg was observed visually by Professor Władysław

Dziewulski in the years 1930-1940 at the vilno observatory, with the $15 \mathrm{~cm}$ refractor using Argelander's method. As a skillful and experienced observer he achieved the accuracy of his single measurement of about 0.05 mag. The series of his 652 estimates of $C H$ Cyg has been recently reduced and is discussed in this paper.

Apparently in those years the star was in relatively quiet state (like in the years 1970-1977), when in the visual region the light of only the red giant component of this binary is visible. We deduce it from:

a) absence of any emission lines in spectra taken by A. Joy in 1930 (Joy 1942)

b) visual brightness of the star almost never exceeding $7.0 \mathrm{mag}$. what is typical for the quiet state of pure M6III spectrum

c) red color, resulting from comparison of our visual brightnesses and the photographic ones published by S. Gaposchkin (1952)

our first conclusion from the analysis of our lightcurve (which cannot be shown here in its full lenght but will be published elsewhere) is that contrary to many previous reports no stable periodicity can be seen. Sometimes only quasiperiods of 100 day or 400 day seem to excite for a cycle or two and disappear.

And yet there is a general sense in this chaotic image. The amplitude of those rather erratic light variations seem to change with the orbital phase of the system. As can be seen in fig.1 the red giant looks the most active near one spectroscopic conjunction and the most quiet near the other. The solid line on the Fig.1 is the cosine of the angle: not companion - red giant - observer, fitted to all the points except of the last six. The orbital parametrs necessary for computing this angle are taken from Mikołajewski, szczerba \& Tomov (this volume) 


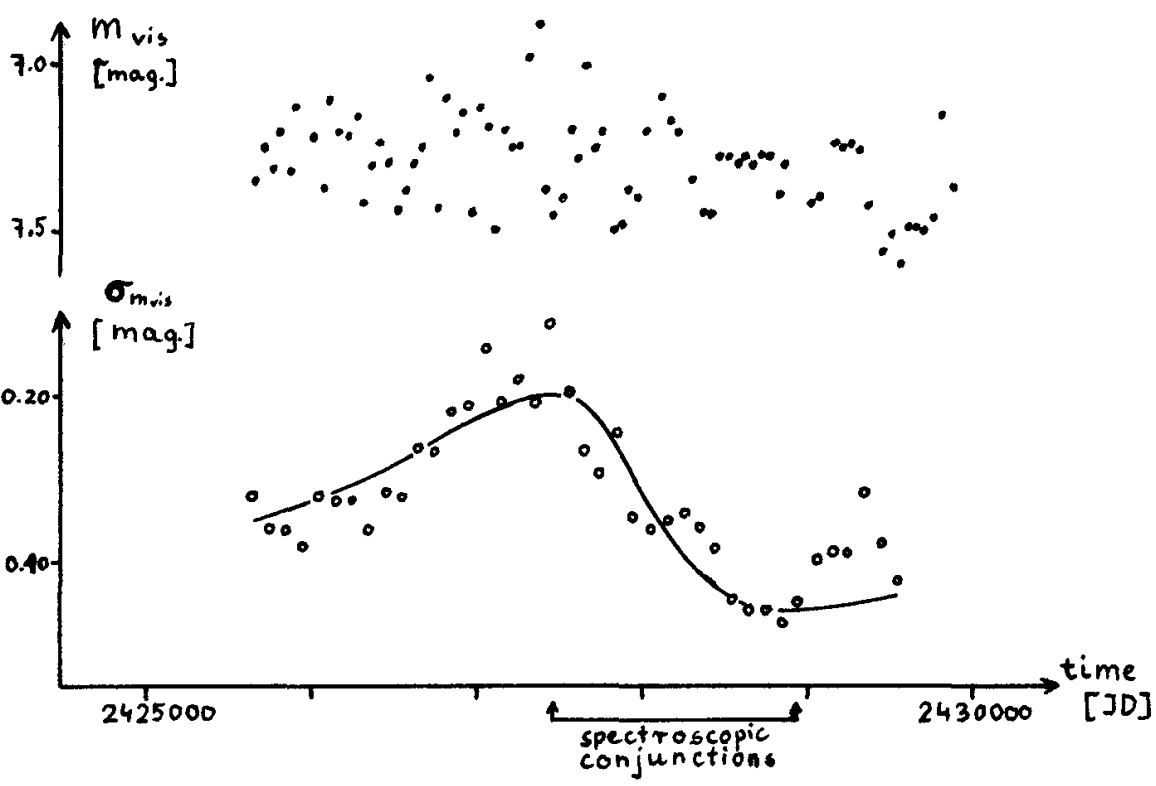

Fig.1 The time variation of the visual brigntness averaged over 50-day intervals (tOD) and dispersion computed for the 300-day intervals, with step of 100 days (bottom).

It seems clear that the observed amplitude of light variations of the red giant follows a function of visibility of the hemisphere facing not companion. it is so for most of the time covered by observations.

Finally, we suggest that in the quiet state of $\mathrm{CH}$ Cyg the hemisphere of the red giant facing the hot companion is more active than the rest of the star. The irradiation by not star induces higher activity on the cool component. What kind of activity could it be? spots? Flares? Nonradial pulsations?

References

1. Gaposchkin S. 1952 Ann. Harvard Obs. 118, 158

2. Joy A. 1942 Astroph. J. 96, 344

*Biographical note

Władysław Dziewulski - professor of astronomy at the Vilno University (1919-1939), later in Torum, the Founding Father of the Nicolaus Copernicus University. He died on

Feb. 5,1962 . 